\title{
Budd-Chiari syndrome
}

United European Gastroenterology Journal 2015, Vol. 3(6) 489-500 (C) Author(s) 2015 Reprints and permissions: sagepub.co.uk/journalsPermissions.nav

Pieter Martens ${ }^{1}$ and Frederik Nevens ${ }^{2}$ DOI: $10.1177 / 2050640615582293$ ueg.sagepub.com

(SAGE

\begin{abstract}
Budd-Chiari syndrome (BCS) is a rare and potentially life-threatening disorder characterized by obstruction of the hepatic outflow tract at any level between the junction of the inferior vena cava with the right atrium and the small hepatic veins. In the West, BCS is a rare hepatic manifestation of one or more underlying prothrombotic risk factors. The most common underlying prothrombotic risk factor is a myeloproliferative disorder, although it is now recognized that almost half of patients have multiple underlying prothrombotic risk factors. Clinical manifestations can be diverse, making BCS a possible differential diagnosis of many acute and chronic liver diseases. The index of suspicion should be very low if there is a known underlying prothrombotic risk factor and new onset of liver disease. Doppler ultrasound is sufficient for confirming the diagnosis, although tomographic imaging (computed tomography (CT) or magnetic resonance imaging (MRI)) is often necessary for further treatment and discussion with a multidisciplinary team. Anticoagulation is the cornerstone of the treatment. Despite the use of anticoagulation, the majority of patients need additional (more invasive) treatment strategies. Algorithms consisting of local angioplasty, TIPS and liver transplantation have been proposed, with treatment choice dictated by a lack of response to a less-invasive treatment regimen. The application of these treatment strategies allows for a five-year survival rate of $90 \%$. In the long term the disease course of BCS can sometimes be complicated by recurrence, progression of the underlying myeloproliferative disorder, or development of post-transplant lymphoma in transplant patients.
\end{abstract}

\section{Keywords}

Budd-Chiari syndrome, treatment, TIPS, liver transplantation, etiology, outcome

Received: 3 January 2015; accepted: 23 March 2015

\section{Introduction}

Budd Chiari syndrome (BCS) is the eponym used for referring to a heterogeneous group of clinical conditions presenting with hepatic venous outflow obstruction. This venous obstruction can be located at any level from the small hepatic veins to the junction of the inferior vena cava (IVC)with the right atrium. ${ }^{1}$ This definition excludes sinusoidal obstruction syndrome and hepatic outflow obstruction secondary to right-sided cardiac disease. BCS is a rare and potentially life-threatening condition. The prevalence of BCS is greatly influenced by geographical differences. While BCS is a more common cause of liver disease in certain Asian countries such as Nepal, it is regarded as a rare disease in Western countries. ${ }^{2}$ In the West the estimated incidence of BCS is one in 2.5 million per person-year. ${ }^{3}$ Geographical variation in anatomical site predilection are also seen between the West and Asia. In the West, thrombosis of the hepatic veins is mostly seen, with less common involvement of the IVC.
In Asia, however, the predominant site of hepatic venous outflow obstruction is the IVC. ${ }^{4-6}$ BCS should be regarded as a hepatic expression of underlying prothrombotic conditions. Geographical differences in these underlying risk factors could explain the variation in anatomical site predilection, with poverty being more common in Asia and oral contraceptive use and myeloproliferative neoplasias being more common in the West. ${ }^{7}$ Irrespective of the cause of hepatic venous outflow obstruction, increased hepatic sinusoidal pressure and portal hypertension quickly ensues, resulting in venous congestion and ischemic damage to the surrounding sinusoidal hepatocytes. ${ }^{8}$ If hepatic sinusoidal

\footnotetext{
${ }^{1}$ Department of Internal Medicine

${ }^{2}$ Department of Liver and Biliopancreatic Disorders, University Hospital Gasthuisberg KU Leuven, Leuven, Belgium

Corresponding author:

Frederik Nevens, Department of Liver and Biliopancreatic Disorders, University Hospital Gasthuisberg KU Leuven, Leuven, Belgium.

Email: frederik.nevens@uzleuven.be
} 
pressure is not relieved by therapeutic interventions or the development of a venous collateral system, then nodular regeneration, fibrosis and ultimately cirrhosis occur. $^{9}$

\section{Clinical manifestation}

The clinical manifestation of BCS is heterogeneous, with presentations ranging from acute liver failure to completely asymptomatic patients. ${ }^{1}$ The classic triad of abdominal pain, ascites and hepatomegaly is commonly present in patients, with abdominal pain presenting in $61 \%$, ascites in $83 \%$ and hepatomegaly in $67 \%$ of patients. ${ }^{3}$ Other clinical features include fever, pedal edema and truncal hepatic veins. Less common clinical manifestations include esophageal bleeding (5\%) and hepatic encephalopathy $(9 \%))^{3} \mathrm{Up}$ to $20 \%$ of patients are completely asymptomatic. ${ }^{10}$ The presentation of BCS depends on the extent and rapidity of hepatic venous outflow obstruction and the presence of decompressing venous collaterals. This concept resulted in classifications of BCS as being fulminant, acute, subacute or chronic. ${ }^{8}$ However, pathological evaluation of affected liver tissue illustrates a dissociation between the acuteness of the clinical presentation and the acuteness of the histological damage. Up to $50 \%$ of patients clinically classified as acute have histological arguments of chronicity (e.g. fibrosis or cirrhosis). ${ }^{11}$ The prognostic value of this clinical classification (fulminant, acute, subacute and chronic) in predicting mortality has not been prospectively validated. ${ }^{12}$ This classification merits little use in clinical practice. To overcome this limitation, several prognostic indices have been designed to predict mortality and response to therapeutic interventions. ${ }^{11,13-15}$ These score systems incorporate clinical and laboratory features to stratify patients, although their use for the management of the individual patient is debatable. ${ }^{16}$

\section{Etiology}

BCS is further classified as being primary or secondary, depending on the exact nature of the hepatic venous outflow obstruction. When flow is obstructed by compression or invasion of a lesion outside the hepatic venous outflow track, it is regarded as being secondary BCS; examples include malignant and cystic extrinsic obstruction. ${ }^{1}$ If flow is obstructed due to an endoluminal aberration, then it is classified as being primary BCS. By far the most common cause of primary BCS is thrombosis, although geographical differences exist with idiopathic membranous obstructions (webs) being prevalent in Asia. ${ }^{17,18}$ In the West, primary BCS can be regarded as being a rare hepatic presentation of an underlying prothrombotic condition. ${ }^{7}$
The underlying prothrombotic condition is mostly undiagnosed when the hepatic venous outflow obstruction presents. Multicenter data illustrate that between $25 \%$ and $46 \%$ of patients have multiple coexisting prothrombotic conditions. ${ }^{3,19,20}$ In contrast to vena porta thrombosis, no intima damage fostering thrombosis is seen in primary BCS in the West. Primary BCS is therefore regarded as a result of a unique constellation of prothrombotic conditions. Table 1 summarizes the prevalence of thrombotic risk factors from a recently published European cohort. ${ }^{3}$ It is worth mentioning that in China the etiology of BCS is different. Data show that prothrombotic disorders are not common in China as a cause of BCS, but endoluminal aberrancies caused by unknown factors (but possibly related to environmental conditions and infection) result in BCS in China. ${ }^{21}$ This review is therefore not applicable to the average Chinese BCS patient.

Myeloproliferative neoplasia (MPN). MPNs are a group of clonal hematological diseases originating from a transformation in a hematopoietic stem cell. Within this group of diseases polycythemia vera, essential thrombocytosis and idiopathic myelofibrosis are collectively referred to as Philadelphia-negative MPNs. ${ }^{22}$ An essential advance in the subject of MPNs was the detection in 2005 of the Janus kinase 2 (JAK2) V617F mutation. $^{23,24}$ This mutation is detected in $90 \%$ of patients

Table 1. Distribution prothrombotic risk factors according to ENVIE Cohort.

\begin{tabular}{lc}
\hline Risk factor & EN-Vie cohort $n / n(\%)^{3}$ \\
\hline Myeloproliferative disorder & $56 / 143(39 \%)$ \\
Inherited thrombophilia & $32 / 154(21 \%)$ \\
- Factor V Leiden & $18 / 147(12 \%)$ \\
- G20210A prothrombin & $5 / 144(3 \%)$ \\
- Protein C deficiency & $5 / 117(4 \%)$ \\
- Protein S deficiency & $3 / 108(3 \%)$ \\
- Antithrombin deficiency & $3 / 112(3 \%)$ \\
Acquired conditions & \\
- Antiphospholipid antibodies & $37 / 150(25 \%)$ \\
- Hyperhomocysteinemia & $28 / 129(22 \%)$ \\
- PNH & $15 / 77(19 \%)$ \\
- Behçet & $4 / 163(2.5 \%)$ \\
- Sarcoïdosis & $2 / 163(1 \%)$ \\
- Oral contraceptives & $31 / 93(33 \%)$ \\
- Pregnancy & $6 / 93(6 \%)$ \\
Combinations & \\
- Single risk factor & $135 / 160(84 \%)$ \\
- Multiple risk factors & $74 / 160(46 \%)$ \\
\hline
\end{tabular}

PNH: paroxysmal nocturnal hemoglobinuria. 
with polycythemia vera and in $50 \%$ of patients with essential thrombocytosis and idiopathic myelofibrosis. $J A K 2$ is a cytoplasmatic tyrosine kinase essential for instigating the intracellular signaling cascade in response to the different growth factor ligands. The $J A K 2 \mathrm{~V} 617 \mathrm{~F}$ mutation results in a constitutive activation of the signal transduction cascade. ${ }^{23,24}$ This mutation is a somatic mutation that can be detected in the peripheral granulocytes or other blood cells originating from the myeloid cell line. ${ }^{22} \mathrm{~A}$ recent retrospective analysis illustrates that detecting $J A K 2 \mathrm{~V} 617 \mathrm{~F}$ mutations is the first diagnostic step for diagnosing an MPN underlying a BCS. ${ }^{25}$ This study also indicates that an underlying MPN confers a more severe form of BCS that can be mitigated by current therapies for BCS. Still a portion of the MPNs do not carry the JAK2 V617F mutation, but carry different mutations. Numerous new mutations are becoming of interest in patients with an MPN, especially in patients with essential thrombocytosis and primary myelofibrosis. Newer mutations including the $J A K 2$ exon 12 , mutation in the thrombopoietin receptor gene, Tet methylcytosine dioxygenase 2 and calreticulin are gaining interest. In the near future the measurement of these somatic mutations will play an important role in the diagnosis and phenotyping of MPN. ${ }^{26-28}$ In situations when there is no detectable mutation, a bone marrow biopsy is necessary to detect the clonal expansion. Using a diagnostic algorithm with detection of the $J A K 2$ V617F mutation first avoids bone marrow biopsies in up to $40 \%$ of patients. ${ }^{25}$ Another important finding is that a normal peripheral blood cell count does not exclude an underlying MPN because hemodilution, hypersplenism and iron deficiency can all contribute to normal peripheral blood cell counts. ${ }^{7}$

Inherited thrombophilia. Inherited thrombophilias are germ line mutations that confer a prothrombotic risk. Most inherited thrombophilias result in increased thrombosis due to an impaired neutralization of thrombin (antithrombin deficiency) or a failure to control the generation of thrombin (Factor V Leiden, protein C deficiency, protein S deficiency and the G20210A prothrombin (PT) gene mutation). ${ }^{29}$ Factor $\mathrm{V}$ Leiden occurs in up to $25 \%$ of patients with BCS. A recent case-control study illustrates that factor V Leiden carries a relative risk of 11.3 for developing $\mathrm{BCS}^{20}$ Still factor V Leiden is almost always accompanied by other prothrombotic risk factors; especially pregnancy appears to be an important multiplicative risk factor. ${ }^{20}$ The $620210 A$ PT gene mutation is a relatively uncommon prothrombotic risk factor $(<5 \%)$ with a weak prothrombotic risk, necessitating that other prothrombotic risk factors be considered as causing BCS. ${ }^{3,19,20}$ The role of protein $\mathrm{C}$, protein $\mathrm{S}$ and antithrombin deficiency is difficult to assess because these anticoagulation factors are produced in the liver and synthesis can be diminished during liver injury. Plasma levels are also diminished in the presence of an acute thrombus. ${ }^{8}$ Because a diagnosis of protein $\mathrm{C}$, protein $\mathrm{S}$ and antithrombin deficiency is based on plasma level measurement of these factors, a diagnosis is often difficult in the acute presentation of BCS (liver injury or active thrombus) but also in the long run when therapy with vitamin $\mathrm{K}$ antagonist is often initiated. Measuring levels of these proteins well below $10 \%-20 \%$ of normal or finding these protein deficiencies in family members argue for an inherited condition. ${ }^{8}$

Acquired conditions. Numerous acquired conditions have been implicated in the development of BCS. Acquired prothrombotic conditions such as Behçet's disease, antiphospholipid syndrome, hyperhomocysteinemia and paroxysmal nocturnal hemoglobinuria (PNH) can contribute to the development of BCS. Behçet's disease is a chronic relapsing systemic inflammatory disease with a high incidence in countries along the silk road (a territorial domain spreading from China to the Mediterranean sea). ${ }^{30}$ When Behçet's disease causes BCS it most often affects the IVC. ${ }^{31,32} \mathrm{PNH}$ is a rare disease that results from a diminished expression of CD55 and CD59, allowing the terminal complement cascade molecule $\mathrm{C} 5$ to tip over from the fluid phase of the complement cascade to the solid phase of complement cascade. Here it creates membrane attack complexes in the absence of the inhibitors CD55 and CD 59. ${ }^{33}$ When PNH causes BCS a liver transplant was generally thought to be contraindicated because of the high chance of recurrence in the liver allograft. However, these data predate the use of the terminal complement inhibitor eculizumab, and reports have been published of successful liver transplantation of PNH with combined use of eculizumab. ${ }^{34}$ Antiphospholipid syndrome is a prothrombotic disorder that can result in venous and arterial thrombosis. Specific autoantibodies are a core feature of antiphospholipid syndrome. ${ }^{35}$ In chronic liver disease anticardiolipin antibodies are often present, complicating the exact determinant of the prevalence of antiphospholipid syndrome in BCS. $^{7}$ Lupus anticoagulant is the antibody associated with the highest risk of developing thrombosis. Documenting this antibody or anti- $\beta 2$-glycoprotein antibody in the face of a hepatic venous outflow obstruction renders the diagnosis of antiphospholipid syndrome. ${ }^{35}$ Using these stringent criteria the estimated prevalence of antiphospholipid syndrome in BCS is between $15 \%$ and $25 \%{ }^{3,7}$ Hyperhomocysteinemia is a state in which excessive homocysteine (catabolic product of methionine) is 
present in the blood. Supraphysiological levels of homocysteine irritate blood vessels and are associated with an increased risk of thrombosis. ${ }^{36}$ Homocysteine is often elevated due to a vitamin deficiency (vitamin B12, vitamin B6 and folate), chronic disease (chronic liver disease, chronic kidney disease, hypothyroidism) or due to a genetic variant in the methylenetetrahydrofolate reductase (MTHFR). The MTHFR mutation has been found to increase the risk of $\mathrm{BCS} ;{ }^{37}$ the exact prevalence of hyperhomocysteinemia is difficult to assess in BCS because homocysteine is often increased during chronic liver disease. ${ }^{38}$ Oral contraceptive use is a frequent risk factor for $\mathrm{BCS} .{ }^{3}$ It carries a relatively mild prothrombotic risk necessitating the consideration of other risk factors. Oral contraceptive use shows a predilection for pure hepatic vein involvement. ${ }^{7}$ Other systemic disease (e.g. sarcoidosis, inflammatory bowel disease (IBD), connective tissue diseases) have been less frequently implicated in the development of BCS. ${ }^{3} \mathrm{~A}$ recent case-control study indicates that a subgroup of patients with an increased clot lysis time are associated with an increased risk for BCS. ${ }^{39}$ The measurement of the activity of the fibrinolytic pathway is currently not a standard in the workup for a patient with BCS. ${ }^{1}$

\section{Diagnostic workup}

An important step in the diagnosis of BCS is to consider the diagnosis in patients presenting with signs or symptoms of hepatic venous outflow obstruction such as painful hepatomegaly, or acute or refractory ascites. The diagnosis should also be considered in any patient with a known prothrombotic condition presenting with acute or chronic liver disease. ${ }^{4}$ The diagnosis of BCS is based on the demonstration of a hepatic venous outflow tract obstruction. This obstruction can be accurately documented by noninvasive imaging such Doppler ultrasonography, computed tomography (CT) or magnetic resonance imaging (MRI). Doppler ultrasonography is regarded as the initial technique of choice and offers a high sensitivity and specificity. ${ }^{40}$ Sonography findings correlate well with pathologic findings or results of invasive imaging. ${ }^{41}$ Table 2 highlights the diagnostic features of BCS on Doppler ultrasonography. CT scan and MRI are imaging modalities that are also efficient in demonstrating a hepatic venous outflow obstruction. MRI is not as effective as Doppler ultrasonography in documenting intrahepatic collaterals, ${ }^{41}$ but MRI and CT are able to better visualize the hepatic parenchyma demonstrating areas of reduced perfusion or necrosis. ${ }^{42}$ MRI and CT offer high spatial resolution, clearly documenting the surrounding anatomy, thereby providing the multidisciplinary team with appropriate images to use for therapeutic planning, such as scheduling a transjugular intrahepatic portosystemic shunt (TIPS). ${ }^{1}$ There are no data available regarding the diagnostic superiority of MRI over CT; however, MRI seems more useful in patients with renal impairment and younger patients given the avoidance of radiation. A liver biopsy is not necessary for diagnosis of BCS. ${ }^{1}$ Because BCS erratically induces parenchyma damage, sampling error is of great concern when performing a biopsy, making biopsy a less effective method for demonstrating the severity of BCS. ${ }^{43}$ Standard laboratory analyses (chemistry panel, blood panel, liver and kidney function tests, international normalized ratio (INR), albumin) offer little help in diagnosing BCS. They do, however, offer insight in predicting the severity of disease, the likelihood of mortality and the possible response to therapy. Albumin, PT or INR, bilirubin, alanine aminotransferase (ALT), and creatinine are laboratory inputs for commonly used prognostic indices in BCS (ChildPugh, model for end-stage liver disease (MELD), Rotterdam index, New Clichy and BCS-TIPS). As mentioned earlier, another important feature of the laboratory workup is the detection of underlying prothrombotic factors. A thorough workup should be undertaken directed at exploring multiple prothrombotic risk factors. The composition of the ascitic fluid is one with a high serum ascites albumin gradient $(\mathrm{SAAG}>1.1 \mathrm{~g} / \mathrm{dl})$ and high protein count $(>2.5 \mathrm{~g} / \mathrm{dl})$, which is similar to that of ascites in cardiac disease. ${ }^{44}$ Table 3 illustrates a complete workup for underlying prothrombotic conditions in BCS.

\section{Treatment}

The natural history of BCS is not well known because there are no cohorts reported of untreated patients. With increasing therapeutic options becoming available over the past decades, overall survival continues to

Table 2. Features specific for hepatic vein obstruction on Doppler ultrasonography. ${ }^{1}$

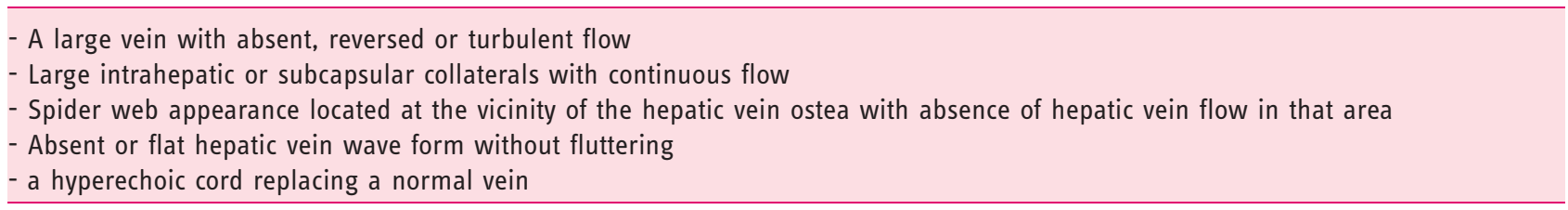


Table 3. Prothrombotic workup newly diagnosed Budd-Chiari patient.

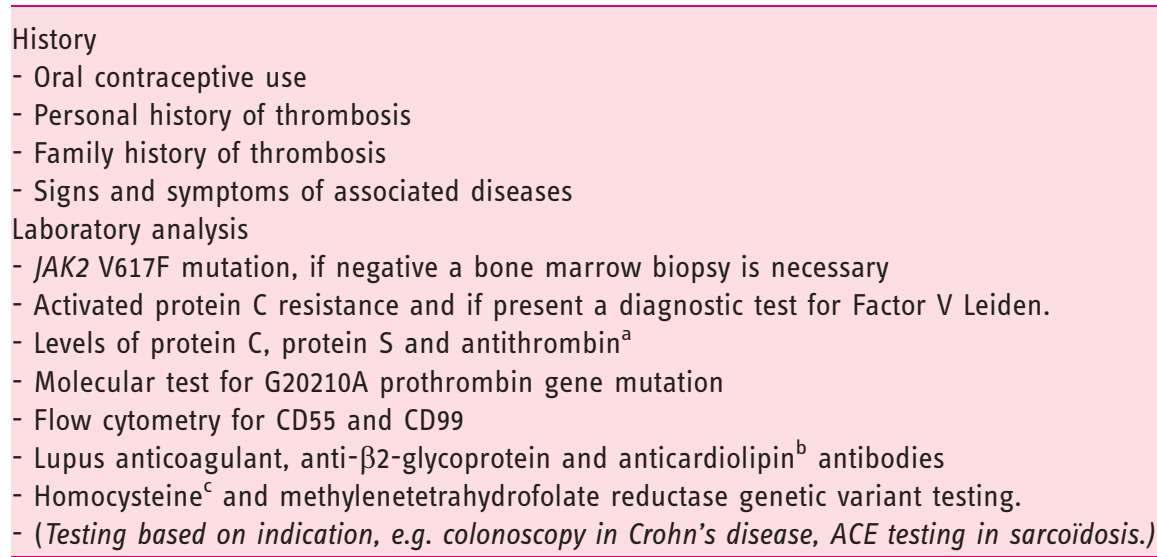

${ }^{a}$ Measurement of protein C, protein $\mathrm{S}$ and antithrombin levels are less useful when marked liver injury is present (documented as an increased international normalized ratio (INR)).

${ }^{b}$ Anticardiolipin antibodies often are presented in states of chronic liver disease and should be carefully interpreted.

'Homocysteine levels are often increased with chronic liver disease. If increased levels of homocysteine are present, underlying vitamin deficiency detection is useful.

JAK2: Janus kinase 2; ACE: angiotensin-converting enzyme.

increase, reaching five-year survival rates between $80 \%$ and $90 \% .3,45-49$ Therapeutic options for BCS include medical management with anticoagulation therapy, decompressing therapies such as recanalization strategies (thrombolytic therapy, stenting and angioplasty) surgical shunting and TIPS and, lastly, orthotopic liver transplantation (OLT). Devoid of randomized, controlled trials (RCTs) comparing one treatment to another, it is impossible for any self-respecting physician to declare one treatment absolutely superior to another. ${ }^{50}$ The rarity of the disease makes it difficult to perform RCTs in patients with BCS. In an effort to continue to improve care, most recommendations regarding treatment are based on case reports, retrospective studies and expert opinions. ${ }^{51}$ Recently a stepwise approach to the treatment of BCS was reported, providing an excellent long-term survival benefit. ${ }^{15}$ A clinical algorithm is proposed in Figure 1.

Medical management. Medical therapy is oriented toward preventing further progression of the venous thrombosis, managing the often pronounced ascites and treating the underlying prothrombotic condition. Anticoagulation should be administered to patients with BCS. ${ }^{1}$ The evidence of using anticoagulation for BCS has been extrapolated from patients presenting with deep vein thrombosis (DVT). In patients with an idiopathic DVT or a DVT with an underlying prothrombotic risk factor that cannot be cured, indefinite anticoagulation is recommended. ${ }^{52}$ A similar strategy is recommended in BCS because of the scarcity of direct evidence in BCS. ${ }^{1}$ One retrospective multivariate analysis in 171 patients of whom $72 \%$ were receiving anticoagulation revealed a nonsignificant trend toward improved survival (relative risk $(R R)=0.14 ; 95 \%$ confidence interval $(\mathrm{CI}), 0.02-$ 1.21 ) in the subgroup of patients with a good prognosis but not in those with intermediate or poor prognoses. ${ }^{45}$ Anticoagulation alone is sufficient in only $10 \%$ of patients, especially those with mild disease. ${ }^{48}$ There are no data to suggest a difference in efficacy of glycosaminoglycans (unfractionated heparin or low-molecular weight heparin (LMWH)) as compared to vitamin K antagonists. ${ }^{7}$ An alarmingly high rate of heparininduced thrombocytopenia (HIT) has been reported with unfractionated heparin in BCS as compared to other indications $(12.3 \%$, compared with $2.7 \%$, respectively). ${ }^{48,53}$ Although HIT has been reported in LMWH use, LMWH should be started promptly in a patient with a new diagnosis of BCS because of its rapid method of action. ${ }^{7}$ Vitamin $\mathrm{K}$ antagonists should be initiated when deemed clinically desirable (no need for quick reversal of the anticoagulant effect) with an INR goal between 2 and 3. ${ }^{1}$ After other therapeutic interventions such as TIPS, surgical portosystemic shunt or liver transplant, anticoagulation will be required. A single retrospective analysis indicates that transplant patients with an underlying MPN well controlled with hydroxyurea benefit from similar antithrombotic effect with aspirin as compared to a vitamin $\mathrm{K}$ antagonist. ${ }^{54} \mathrm{In}$ DVT practice it is often common for female patients to continue the use of oral contraceptives while receiving anticoagulation. The discontinuation of oral contraceptives during anticoagulation results in heavy menstrual bleeding. Although no direct data exist, similar practice is probably possible in BCS patients. Advice for the management of portal hypertension-related complications in BCS is similar to that for patients with cirrhosis. ${ }^{55}$ 


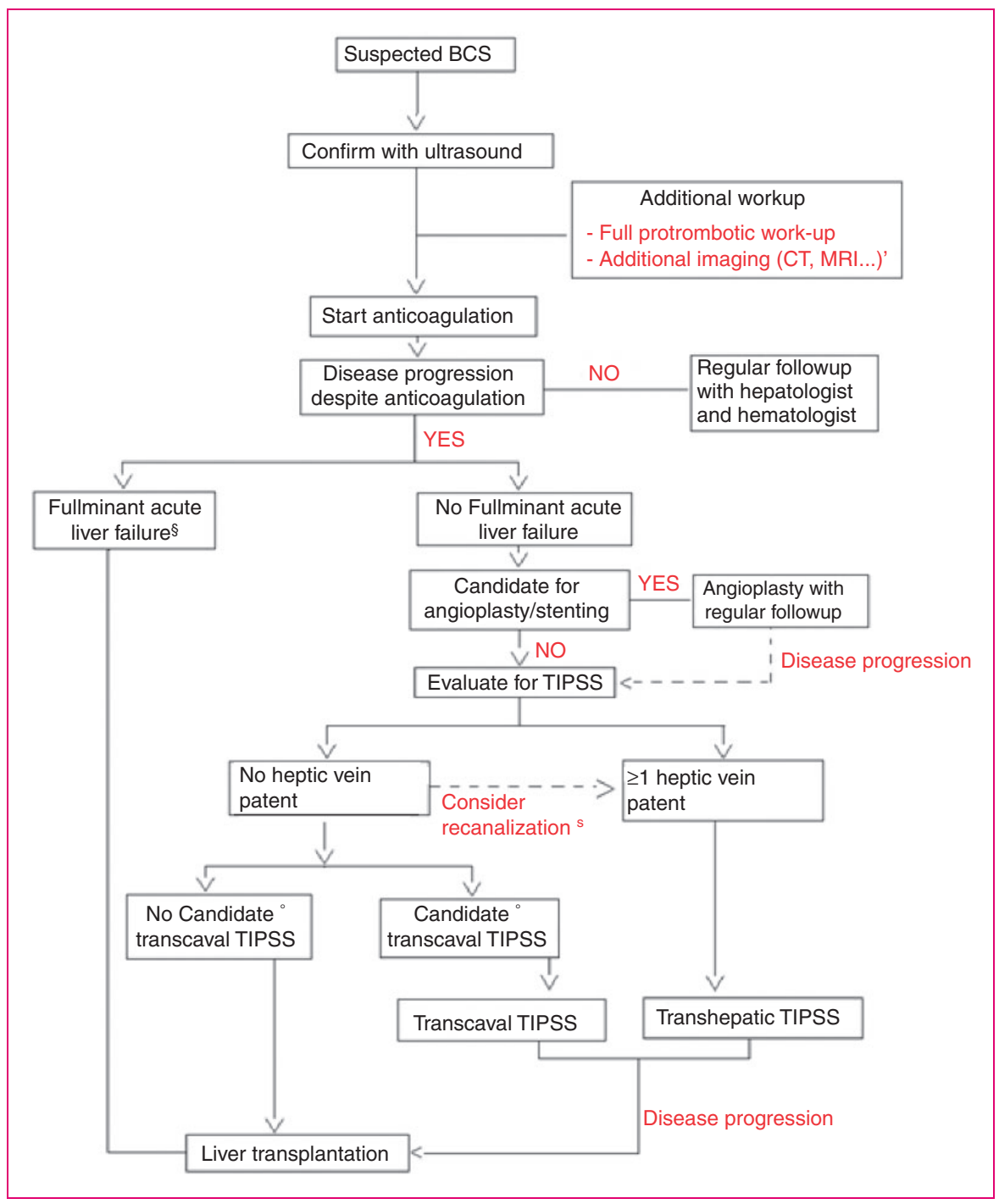

Figure 1. A proposed diagnostic and therapeutic work-up for the patient with Budd Chiari syndrome (BCS).

*Additional imaging after duplex diagnosis is required for future therapeutic planning and for discussion with the multidisciplinary team (evaluation possibility of angioplasty, TIPS or liver transplantation.)

§If the patient presents with acute fulminant liver failure (or BCS-TIPS score $>7$ ) immediate referral for liver transplantation seems feasible as first option.

- Suitability for transcaval TIPS depends on the experience of the center with transcaval TIPS.

$\$$ If no hepatic veins are patent but thrombus is fresh, recanalization of the hepatic veins can first be tried if successful TIPS placement can be performed by a transhepatic route.

CT: computed tomography; MRI: magnetic resonance imaging; TIPS: transjugular intrahepatic portosystemic shunt.

Decompressive therapies. Decompressive therapies consist of Thrombolytic therapy and angioplasty, Surgical portosystemic shunt and TIPS. OLT however is not part of the decompressive therapies and is a treatment section on its own. such as decompressive therapies and medical management. Thus:

1. medical management

2. decompressive therapies with subsection

- Thrombolytic therapy and angioplasty

- Surgical portosystemic shunt

\section{- TIPS}

\section{OLT}

Thrombolytic therapy and angioplasty. Thrombolytic therapy offers little benefit in clinical practice. The limited amount of available data on thrombolytic therapy found the benefit to be inconclusive. ${ }^{56}$ The rationale of angioplasty is to decompress the liver while restoring hepatic blood flow. Luminal strategies to restore blood flow include balloon angioplasty with or without 
subsequent stenting. Patients with focal or segmental obstructions of the hepatic outflow tract are theoretically eligible for angioplasty. Short-length stenosis presents in $25 \%{ }^{57}$ of patients with pure hepatic vein block and up to $60 \%$ of patients with an IVC block. ${ }^{58}$ The experience with angioplasty is extensive for patients presenting with an IVC block, with more than 453 patients being reported. ${ }^{1}$ The technical success rate is high, in the $95 \%$ range with an almost instant post-procedural improvement in signs, symptoms and liver function. ${ }^{1}$ Angioplasty of pure hepatic vein block has been less reported. ${ }^{59-64}$ The overall success rate is unclear. In general re-obstruction is more common in patients undergoing balloon angioplasty as compared to stenting. Severe complications occur seldom and rescue liver transplantation was not necessary. Stent migration toward the right atrium has been described, and the choice of stenting versus balloon angioplasty largely depends on local experience. Still, it should be mentioned that patients presenting with a single shortlength stenosis of a hepatic vein, if left untreated, have a better outcome than patients with multiple hepatic vein involvement. ${ }^{57}$ Still it is probably worthwhile to screen patients for focal or segmental obstructions of the hepatic outflow tract. ${ }^{15}$

Surgical portosystemic shunt. The therapeutic principle of a portosystemic shunt is to convert the portal vein into an outflow tract, thereby decompressing the hepatic sinusoids. Surgical portosystemic shunts have now been almost completely abandoned as a therapeutic option, as illustrated by a follow-up of the ENVie cohort showing that not a single patient received a portosystemic shunt in the follow-up period. ${ }^{15}$ The observation that perioperative mortality is $25 \%{ }^{65}$ and late shunt patency is only $70 \%{ }^{66}$ could account for this finding. Surgical portosystemic shunting can also be technically difficult when there is caudate lobe hypertrophy. ${ }^{67}$ Surgical portosystemic shunting failed to show a benefit of survival in four multicenter retrospective multivariate analyses after adjusting for independent prognostic factors. ${ }^{11,43,45,68}$ This consistent inability of surgical portosystemic shunting to show a survival benefit resulted in this treatment option falling out of favor. ${ }^{15}$

TIPS. TIPS is a percutaneous image-guided procedure that creates a portocaval shunt that passes through the liver parenchyma. ${ }^{69}$ TIPS has gained wide acceptance (level IA evidence) as a valuable treatment option for secondary prevention of a variceal bleed ${ }^{70,71}$ and refractory ascites. ${ }^{72,73}$ TIPS patency has also significantly improved since the use of polytetrafluoroethylene (PTFE)-covered stents. ${ }^{74}$ Recently TIPS has been reported as being a successful treatment option in
BCS. ${ }^{14,75}$ A TIPS procedure is technically more challenging in a patient with BCS as compared to cirrhosis. In experienced centers the success rate of TIPS placement is $80 \%$ in BCS as compared to greater than $90 \%$ for cirrhosis. $^{2,76}$ Given the drawbacks of portosystemic shunting, TIPS seems an attractive alternative to decompress the liver, especially since only $18 \%$ of patients are well controlled with medical treatment alone. $^{48}$ When adding recanalization therapies (thrombolytic therapy, angioplasty or stenting) of short-length stenosis to the medical treatment, a total of $66.5 \%$ of patients were still not adequately managed. ${ }^{14}$ These patients are theoretical candidates for TIPS placement. A large European retrospective multicenter study performed in highly experienced TIPS centers involving 124 patients showed improved survival especially in high-risk patients (Rotterdam classification III). ${ }^{14}$ The expected five-year OLT-free survival in the high-risk patients was estimated at $42 \%$ but increased to $71 \%$ after TIPS placement. ${ }^{14}$ This fiveyear OLT-free survival correlates well with the survival of similar BCS patients who underwent transplantation. ${ }^{77,78}$ A more recently published United Kingdom (UK)-based retrospective study confirms the findings that TIPS results in a good long-term outcome, and TIPS patency is excellent when using PTFEcovered stents. ${ }^{79}$ These combined data illustrate that TIPS should be considered first in the patient failing medical management and recanalization therapies before proceeding to OLT. It should be pointed out that the centers performing TIPS in this study had high levels of experience with placing TIPS in BCS patients, resulting in a success rate of $93 \%$ of placement. The European retrospective study identified a subgroup with an extraordinarily bad baseline prognosis (defined as BCS-TIPS > 7) probably benefiting more from early OLT instead of a treatment trial with TIPS. ${ }^{70}$ The UKbased retrospective study confirms that a high BCSTIPS score is associated with a significant increase in mortality, but negates the use of other prognostic indices. ${ }^{79}$ These extremely high-risk patients often have hepatic encephalopathy, thus presenting with fulminant hepatic failure from the onset. ${ }^{15} \mathrm{~A}$ recent follow-up of the EN-Vie cohort illustrated that with this stepwise management up to $70 \%$ of invasive-treated patients received TIPS and only $22 \%$ received OLT. ${ }^{15}$

OLT. OLT is regarded as the salvage therapy when other less-invasive therapies fail and a treatment of choice for those presenting with fulminant hepatic failure from the beginning. ${ }^{15,80}$ About $10 \%-20 \%$ of patients show progressive liver deterioration despite medical management, revascularization strategies and TIPS. In these patients an OLT is the only remaining therapy. Several large retrospective analyses have 
evaluated the benefit of OLT in BCS on survival, ${ }^{77,78,81}$ reporting five-year survival rates varying between $71 \%$ and $89.4 \%$. Variation between survival rates can be explained by the difference in baseline characteristics of included patients. In a large European study involving 248 patients, more than $50 \%$ of patients were Rotterdam class III. ${ }^{78}$ In this group the five-year survival was $71 \%$, which is clearly superior to the $42 \%$ five-year rated survival without invasive therapy. ${ }^{45,78}$ The study by Ulrich et al. included patients with milder disease as characterized by $69 \%$ of patients having a Child-Pugh score A or B. In this study the five-year survival rate was $89.4 \%$, which does not differ that much from the survival rate of an untreated Child-Pugh class A. ${ }^{77,82}$ This indicates that the survival benefit of OLT is most pronounced in those BCS patients with worse baseline characteristics. The survival rate and graft function after OLT in BCS patients are similar ${ }^{78}$ or even superior ${ }^{77}$ to those transplanted for other indications. A previous TIPS placement is not associated with a poor outcome after OLT. ${ }^{81}$ Early initiation and persistent anticoagulation after OLT is suggested as having a favorable impact on survival., ${ }^{4}, 7$ This is illustrated by a European study that had similar rates of bleeding and thrombosis (both $11 \%$ ), but the mortality associated with bleeding was $1 \%$ while the mortality after thrombosis was $40.7 \%$. As mentioned earlier, aspirin associated with hydroxyurea resulted in the same outcome as vitamin $\mathrm{K}$ antagonists after OLT for BCS, when the underlying prothrombotic cause was an MPN. ${ }^{54}$ Still it should be underscored that a growing body of evidence indicates that BCS is the result of numerous prothrombotic conditions, suggesting that this practice could carry some risk, especially if a screening for prothrombotic conditions was not fully complete. There is no evidence that the use of immunosuppressives for OLT causes an accelerated malignant transformation of an underlying MPN. ${ }^{2}$

\section{Prognostic indices}

The induction of comparability between studies by different centers is essential for making valid conclusions in rare diseases. To induce comparability, baseline characteristics are often translated into prognostic indices. These scores stratify patients into subgroups for which therapeutics decisions and prognoses might differ. This strategy is necessary because RCTs are impossible to conduct in BCS because of its rarity. Several scores are evaluated in BCS such as the Child-Pugh, MELD, Clichy PI, Rotterdam score, New Clichy PI and BCS-TIPS. ${ }^{16}$ Although these scores are useful for comparing large cohorts, $3,15,45$ they lack the prognostic accuracy for managing the individual patient. ${ }^{16}$ When these prognostic indices were evaluated for OLT-free survival in an independent cohort, they all showed an area under receiver-operating characteristic curves (AUROCs) of less than $0.70 .{ }^{16}$ Generally prognostic indices with AUROCs between 0.80 and 0.90 are deemed prognostic for managing the individual patient. ${ }^{83}$ So in clinical practice the choice of therapy should probably be based on the lack of response to a lesser invasive therapy. Although there are no formal criteria for stepping up therapy, some criteria have been described such as persistence of symptoms such as ascites despite treatment, presence of debilitating encephalopathy or progressive deterioration of liver function tests. ${ }^{15}$

\section{Follow-up}

Current guidelines advocate the systematic follow-up of patients diagnosed with BCS. ${ }^{1}$ Yet few data are available on the optimal timing and methods of follow-up. Patients should be monitored for disease progression, although no uniform definition of disease progression exists. In patients with cirrhosis secondary to BCS, the patient should be followed up in a similar fashion as cirrhosis due to other causes. ${ }^{55}$ Because the majority of the patients have an underlying MPN or other prothrombotic condition, regular follow-up should be scheduled with a hematologist. To date no data exist to support an accelerated disease trajectory of the underlying MPN toward acute myeloid leukemia in BCS patients with an underlying MPN treated with OLT. The few data available suggest that pregnancy is possible in patients with BCS. Maternal outcome is good; fetal outcome is most often dictated by the underlying prothrombotic conditions. ${ }^{84}$ It is therefore advisable that pregnant women with a history of BCS receive care in a specialized center with expertise available. The occurrence of benign macro-regenerative liver nodules is common in diseases that manifest with hepatic venous outflow obstruction (BCS, status postLa Fontaine surgery, pericarditis constriva). ${ }^{85}$ The occurrence of hepatocellular carcinoma (HCC) in BCS patients has also been well documented. A French study indicates that up to $11.3 \%$ of BCS patients develop an HCC during a period of 18 years' follow-up. ${ }^{86} \mathrm{~A}$ recent systematic review indicates that the hepatic venous pressure gradient is the most important risk factor for the development of HCC. ${ }^{87}$ The differentiation between benign macro-regenerative nodules and HCC is difficult. ${ }^{1}$ A rising alphafetoprotein carries a high specificity but lacks diagnostic sensitivity. ${ }^{88,89}$ More recent data indicate that MRI allows for the detection of specific patterns of HCC in BCS patients. ${ }^{90}$ To date no consensus exists about the optimal screening interval and at what timing imaging modalities should be used. Currently most 
centers apply a yearly follow-up with ultrasound and measurement of alpha-fetoprotein. When hepatic nodules occur, MRI or liver biopsy is most often used to differentiate benign regenerative nodules from HCC.

\section{Future research}

Recent multicenter work has resulted in an increased understanding of BCS and improved outcomes. Continued effort needs to be made in pooling patients and deciding which patients are good candidates for certain therapies. Few data are available on the use of novel anticoagulants (rivaroxaban, apixaban and dabigatran) in BCS. Efforts should be made in addressing the definition of disease progression under certain therapies. Further research is needed to determine the precise role of MRI for differentiating benign liver nodules with $\mathrm{HCC}$ in BCS patients.

\section{Conclusion}

BCS is a rare and potentially life-threatening condition associated with hepatic venous outflow obstruction. In the West, thrombosis is the main reason for hepatic outflow obstruction. It is currently clear that most patients have multiple underlying prothrombotic conditions, underscoring the importance of a thorough workup. Some prothrombotic factors foster a predilection for occurring in a certain area of the hepatic venous outflow tract (e.g. pure hepatic involvement versus IVC involvement.) In the West there is a predominance of prothrombotic conditions affecting purely the hepatic veins. Doppler ultrasonography is often sufficient for documenting hepatic venous outflow tract obstruction. However, when choosing a treatment strategy among a multidisciplinary team of hepatologists, interventional radiologists and transplant surgeons, other imaging such as CT and MRI is most often necessary. All patients should be started on anticoagulation given the presence of underlying prothrombotic conditions. Still, anticoagulation is sufficient in only $18 \%$ of patients in halting the progression of liver disease, often necessitating more-invasive treatment strategies. When imaging reveals a short focal and segmental stenosis within the hepatic outflow tract, recanalization with angioplasty and stenting seems reasonable. However, most patients will have a more diffuse affliction within the hepatic outflow tract. For these patients TIPS is a suitable solution. TIPS efficiently decompresses the liver parenchyma, and has a clear impact on survival. Currently in Europe up to $70 \%$ of invasively treated BCSs are effectively treated with TIPS. If despite TIPS there is progressive liver injury, then patients should be treated with OLT. For the Budd-Chiari patient presenting with fulminant hepatic failure, first-line treatment with OLT seems reasonable. It is clear that for these reasons patients with BCS should be treated in a center able to perform OLT. ${ }^{2}$

\section{Funding}

This research received no specific grant from any funding agency in the public, commercial, or not-for-profit sectors.

\section{Conflict of interest}

None declared.

\section{References}

1. DeLeve LD, Valla DC, Garcia-Tsao G, et al. Vascular disorders of the liver. Hepatology 2009; 49: 1729-1764.

2. Plessier A and Valla DC. Budd-Chiari syndrome. Semin Liver Dis 2008; 28: 259-269.

3. Darwish MS, Plessier A, Hernandez-Guerra M, et al. Etiology, management, and outcome of the BuddChiari syndrome. Ann Intern Med 2009; 151: 167-175.

4. Hoekstra J and Janssen HL. Vascular liver disorders (I): Diagnosis, treatment and prognosis of Budd-Chiari syndrome. Neth J Med 2008; 66: 334-339.

5. Qi X, Zhang C, Han G, et al. Prevalence of the $J A K 2 \mathrm{~V} 617 \mathrm{~F}$ mutation in Chinese patients with BuddChiari syndrome and portal vein thrombosis: A prospective study. J Gastroenterol Hepatol 2012; 27: 1036-1043.

6. Qi X, He C, Han G, et al. Prevalence of paroxysmal nocturnal hemoglobinuria in Chinese patients with Budd-Chiari syndrome or portal vein thrombosis. J Gastroenterol Hepatol 2013; 28: 148-152.

7. Valla DC. Primary Budd-Chiari syndrome. J Hepatol 2009; 50: 195-203.

8. Menon KV, Shah V and Kamath PS. The Budd-Chiari syndrome. $N$ Engl J Med 2004; 350: 578-585.

9. Cazals-Hatem D, Vilgrain V, Genin P, et al. Arterial and portal circulation and parenchymal changes in BuddChiari syndrome: A study in 17 explanted livers. Hepatology 2003; 37: 510-519.

10. Hadengue A, Poliquin M, Vilgrain V, et al. The changing scene of hepatic vein thrombosis: Recognition of asymptomatic cases. Gastroenterology 1994; 106: 1042-1047.

11. Langlet P, Escolano S, Valla D, et al. Clinicopathological forms and prognostic index in Budd-Chiari syndrome. J Hepatol 2003; 39: 496-501.

12. Senzolo M, Cholongitas EC, Patch D, et al. Update on the classification, assessment of prognosis and therapy of Budd-Chiari syndrome. Nat Clin Pract Gastroenterol Hepatol 2005; 2: 182-190.

13. Darwish MS, Kim WR, de Groen PC, et al. Can the model for end-stage liver disease be used to predict the prognosis in patients with Budd-Chiari syndrome? Liver Transpl 2007; 13: 867-874.

14. García-Pagán JC, Heydtmann M, Raffa S, et al. TIPS for Budd-Chiari syndrome: Long-term results and 
prognostics factors in 124 patients. Gastroenterology 2008; 135: 808-815.

15. Seijo S, Plessier A, Hoekstra J, et al. Good long-term outcome of Budd-Chiari syndrome with a step-wise management. Hepatology 2013; 57: 1962-1968.

16. Rautou PE, Moucari R, Escolano S, et al. Prognostic indices for Budd-Chiari syndrome: Valid for clinical studies but insufficient for individual management. Am J Gastroenterol 2009; 104: 1140-1146.

17. Al Himali SH, Al Osaimi AM, Dahab ST, et al. Budd Chiari syndrome: Experience at King Faisal Specialist Hospital and Research Centre, Riyadh, Saudi Arabia. Saudi J Gastroenterol 2003; 9: 11-14.

18. De BK, De KK, Sen S, et al. Etiology based prevalence of Budd-Chiari syndrome in eastern India. $J$ Assoc Physicians India 2000; 48: 800-803.

19. Denninger MH, Chait Y, Casadevall N, et al. Cause of portal or hepatic venous thrombosis in adults: The role of multiple concurrent factors. Hepatology 2000; 31: 587-591.

20. Janssen HL, Meinardi JR, Vleggaar FP, et al. Factor V Leiden mutation, prothrombin gene mutation, and deficiencies in coagulation inhibitors associated with BuddChiari syndrome and portal vein thrombosis: Results of a case-control study. Blood 2000; 96: 2364-2368.

21. Dang X, Li L and Xu P. Research status of Budd-Chiari syndrome in China. Int J Clin Exp Med 2014; 7: 4646-4652.

22. Campbell PJ and Green AR. The myeloproliferative disorders. $N$ Engl J Med 2006; 355: 2452-2466.

23. Baxter EJ, Scott LM, Campbell PJ, et al. Acquired mutation of the tyrosine kinase $J A K 2$ in human myeloproliferative disorders. Lancet 2005; 365: 1054-1061.

24. James C, Ugo V, Le Couedic JP, et al. A unique clonal $J A K 2$ mutation leading to constitutive signalling causes polycythaemia vera. Nature 2005; 434: 1144-1148.

25. Kiladjian JJ, Cervantes F, Leebeek FW, et al. The impact of $J A K 2$ and $M P L$ mutations on diagnosis and prognosis of splanchnic vein thrombosis: A report on 241 cases. Blood 2008; 111: 4922-4929.

26. Klampfl T, Gisslinger H, Harutyunyan AS, et al. Somatic mutations of calreticulin in myeloproliferative neoplasms. $N$ Engl J Med 2013; 369: 2379-2390.

27. Ortmann CA, Kent DG, Nangalia J, et al. Effect of mutation order on myeloproliferative neoplasms. $N$ Engl $J$ Med 2015; 372: 601-612.

28. Rumi E, Pietra D, Guglielmelli P, et al. Acquired copyneutral loss of heterozygosity of chromosome $1 \mathrm{p}$ as a molecular event associated with marrow fibrosis in MPL-mutated myeloproliferative neoplasms. Blood 2013; 121: 4388-4395.

29. Seligsohn U and Lubetsky A. Genetic susceptibility to venous thrombosis. $N$ Engl J Med 2001; 344: $1222-1231$.

30. Li HZ, Guo J, Lin H, et al. Silk road occlusion. Lancet 2011; 377: 1046.

31. Bayraktar Y, Balkanci F, Bayraktar M, et al. BuddChiari syndrome: A common complication of Behçet's disease. Am J Gastroenterol 1997; 92: 858-862.
32. Bismuth E, Hadengue A, Hammel P, et al. Hepatic vein thrombosis in Behçet's disease. Hepatology 1990; 11 : 969-974.

33. Hill A, Kelly RJ and Hillmen P. Thrombosis in paroxysmal nocturnal hemoglobinuria. Blood 2013; 121: 4985-4996.

34. Singer AL, Locke JE, Stewart ZA, et al. Successful liver transplantation for Budd-Chiari syndrome in a patient with paroxysmal nocturnal hemoglobinuria treated with the anti-complement antibody eculizumab. Liver Transpl 2009; 15: 540-543.

35. Giannakopoulos B and Krilis SA. The pathogenesis of the antiphospholipid syndrome. N Engl J Med 2013; 368: 1033-1044.

36. Varga EA, Sturm AC, Misita CP, et al. Cardiology patient pages. Homocysteine and MTHFR mutations: Relation to thrombosis and coronary artery disease. Circulation 2005; 111: e289-e293.

37. Li XM, Wei YF, Hao HL, et al. Hyperhomocysteinemia and the MTHFR C677T mutation in Budd-Chiari syndrome. Am J Hematol 2002; 71: 11-14.

38. Shetty $\mathrm{S}$ and Ghosh K. Thrombophilic dimension of Budd Chiari syndrome and portal venous thrombosis - a concise review. Thromb Res 2011; 127: 505-512.

39. Hoekstra J, Guimaraes AH, Leebeek FW, et al. Impaired fibrinolysis as a risk factor for Budd-Chiari syndrome. Blood 2010; 115: 388-395.

40. Bolondi L, Gaiani S, Li BS, et al. Diagnosis of BuddChiari syndrome by pulsed Doppler ultrasound. Gastroenterology 1991; 100 (5 Pt 1): 1324-1331.

41. Miller WJ, Federle MP, Straub WH, et al. Budd-Chiari syndrome: Imaging with pathologic correlation. Abdom Imaging 1993; 18: 329-335.

42. Buckley O, O'Brien J, Snow A, et al. Imaging of BuddChiari syndrome. Eur Radiol 2007; 17: 2071-2078.

43. Tang TJ, Batts KP, de Groen PC, et al. The prognostic value of histology in the assessment of patients with Budd-Chiari syndrome. J Hepatol 2001; 35: 338-343.

44. Chung RT, Iafrate AJ, Amrein PC, et al. Case records of the Massachusetts General Hospital. Case 15-2006. A 46year-old woman with sudden onset of abdominal distention. $N$ Engl J Med 2006; 354: 2166-2175.

45. Darwish MS, Valla DC, de Groen PC, et al. Determinants of survival and the effect of portosystemic shunting in patients with Budd-Chiari syndrome. Hepatology 2004; 39: 500-508.

46. Eapen CE, Velissaris D, Heydtmann M, et al. Favourable medium term outcome following hepatic vein recanalisation and/or transjugular intrahepatic portosystemic shunt for Budd Chiari syndrome. Gut 2006; 55: 878-884.

47. Perelló A, García-Pagán JC, Gilabert R, et al. TIPS is a useful long-term derivative therapy for patients with Budd-Chiari syndrome uncontrolled by medical therapy. Hepatology 2002; 35: 132-139.

48. Plessier A, Sibert A, Consigny Y, et al. Aiming at minimal invasiveness as a therapeutic strategy for BuddChiari syndrome. Hepatology 2006; 44: 1308-1316.

49. Rossle M, Olschewski M, Siegerstetter V, et al. The Budd-Chiari syndrome: Outcome after treatment 
with the transjugular intrahepatic portosystemic shunt. Surgery 2004; 135: 394-403.

50. Collier R. Legumes, lemons and streptomycin: A short history of the clinical trial. CMAJ 2009; 180: 23-24.

51. Janssen HL, García-Pagán JC, Elias E, et al. Budd-Chiari syndrome: A review by an expert panel. J Hepatol 2003; 38: 364-371.

52. Bates SM and Ginsberg JS. Clinical practice. Treatment of deep-vein thrombosis. $N$ Engl J Med 2004; 351: 268-277.

53. Warkentin TE, Levine MN, Hirsh J, et al. Heparininduced thrombocytopenia in patients treated with lowmolecular-weight heparin or unfractionated heparin. N Engl J Med 1995; 332: 1330-1335.

54. Chinnakotla S, Klintmalm GB, Kim P, et al. Long-term follow-up of liver transplantation for Budd-Chiari syndrome with antithrombotic therapy based on the etiology. Transplantation 2011; 92: 341-345.

55. Runyon BA. Management of adult patients with ascites caused by cirrhosis. Hepatology 1998; 27: 264-272.

56. Sharma S, Texeira A, Texeira P, et al. Pharmacological thrombolysis in Budd Chiari syndrome: A single centre experience and review of the literature. J Hepatol 2004; 40: $172-180$.

57. Valla D, Hadengue A, el Younsi M, et al. Hepatic venous outflow block caused by short-length hepatic vein stenoses. Hepatology 1997; 25: 814-819.

58. Okuda K. Inferior vena cava thrombosis at its hepatic portion (obliterative hepatocavopathy). Semin Liver Dis 2002; 22: 15-26.

59. Griffith JF, Mahmoud AE, Cooper S, et al. Radiological intervention in Budd-Chiari syndrome: Techniques and outcome in 18 patients. Clin Radiol 1996; 51: 775-784.

60. Martin LG, Henderson JM, Millikan WJ Jr, et al. Angioplasty for long-term treatment of patients with Budd-Chiari syndrome. AJR Am J Roentgenol 1990; 154: 1007-1010.

61. Núñez $O$, de la Cruz G, Molina J, et al. Interventional radiology, angioplasty and TIPS in Budd-Chiari syndrome [article in Spanish]. Gastroenterol Hepatol 2003; 26: 461-464.

62. Pelage JP, Denys A, Valla D, et al. Budd-Chiari syndrome due to prothrombotic disorder: Mid-term patency and efficacy of endovascular stents. Eur Radiol 2003; 13 : 286-293.

63. Qiao T, Liu CJ, Liu C, et al. Interventional endovascular treatment for Budd-Chiari syndrome with long-term follow-up. Swiss Med Wkly 2005; 135: 318-326.

64. Witte AM, Kool LJ, Veenendaal R, et al. Hepatic vein stenting for Budd-Chiari syndrome. Am J Gastroenterol 1997; 92: 498-501.

65. Langlet $\mathrm{P}$ and Valla D. Is surgical portosystemic shunt the treatment of choice in Budd-Chiari syndrome? Acta Gastroenterol Belg 2002; 65: 155-160.

66. Panis Y, Belghiti J, Valla D, et al. Portosystemic shunt in Budd-Chiari syndrome: Long-term survival and factors affecting shunt patency in 25 patients in Western countries. Surgery 1994; 115: 276-281.

67. Park JS, Federle MP and Sass DA. Education and imaging. Hepatobiliary and pancreatic: Budd-Chiari syndrome presenting as a caudate lobe pseudotumor. J Gastroenterol Hepatol 2010; 25: 219.

68. Zeitoun G, Escolano S, Hadengue A, et al. Outcome of Budd-Chiari syndrome: A multivariate analysis of factors related to survival including surgical portosystemic shunting. Hepatology 1999; 30: 84-89.

69. Fidelman N, Kwan SW, Laberge JM, et al. The transjugular intrahepatic portosystemic shunt: An update. AJR Am J Roentgenol 2012; 199: 746-755.

70. García-Pagán JC, Caca K, Bureau C, et al. Early use of TIPS in patients with cirrhosis and variceal bleeding. $N$ Engl J Med 2010; 362: 2370-2379.

71. Rössle M, Haag K, Ochs A, et al. The transjugular intrahepatic portosystemic stent-shunt procedure for variceal bleeding. N Engl J Med 1994; 330: 165-171.

72. Rössle M. The transjugular intrahepatic portosystemic shunt. J Hepatol 1996; 25: 224-231.

73. Rössle M, Ochs A, Gulberg V, et al. A comparison of paracentesis and transjugular intrahepatic portosystemic shunting in patients with ascites. $N$ Engl J Med 2000; 342: 1701-1707.

74. Hernández-Guerra $\mathrm{M}$, Turnes $\mathrm{J}$, Rubinstein $\mathrm{P}$, et al. PTFE-covered stents improve TIPS patency in BuddChiari syndrome. Hepatology 2004; 40: 1197-1202.

75. Fitsiori K, Tsitskari M, Kelekis A, et al. Transjugular intrahepatic portosystemic shunt for the treatment of Budd-Chiari syndrome patients: Results from a single center. Cardiovasc Intervent Radiol 2014; 37: 691-697.

76. Boyer TD and Haskal ZJ. The role of transjugular intrahepatic portosystemic shunt (TIPS) in the management of portal hypertension: Update 2009. Hepatology 2010; 51: 306.

77. Ulrich F, Pratschke J, Neumann U, et al. Eighteen years of liver transplantation experience in patients with advanced Budd-Chiari syndrome. Liver Transpl 2008; 14: 144-150.

78. Mentha G, Giostra E, Majno PE, et al. Liver transplantation for Budd-Chiari syndrome: A European study on 248 patients from 51 centres. J Hepatol 2006; 44: 520-528.

79. Tripathi D, Macnicholas R, Kothari C, et al. Good clinical outcomes following transjugular intrahepatic portosystemic stent-shunts in Budd-Chiari syndrome. Aliment Pharmacol Ther 2014; 39: 864-872.

80. Senzolo M, Cholongitas EC, Patch D, et al. Update on the classification, assessment of prognosis and therapy of Budd-Chiari syndrome. Nat Clin Pract Gastroenterol Hepatol 2005; 2: 182-190.

81. Segev DL, Nguyen GC, Locke JE, et al. Twenty years of liver transplantation for Budd-Chiari syndrome: A national registry analysis. Liver Transpl 2007; 13: 1285-1294.

82. Darwish MS and Kamath PS. Liver transplantation for Budd-Chiari syndrome: When is it really necessary? Liver Transpl 2008; 14: 133-135.

83. Kamath PS, Wiesner RH, Malinchoc M, et al. A model to predict survival in patients with end-stage liver disease. Hepatology 2001; 33: 464-470. 
84. Aggarwal N, Suri V, Chopra S, et al. Pregnancy outcome in Budd Chiari syndrome - a tertiary care centre experience. Arch Gynecol Obstet 2013; 288: 949-952.

85. Newerla C, Schaeffer F, Terracciano L, et al. Multiple FNH-like lesions in a patient with chronic Budd-Chiari syndrome: Gd-EOB-enhanced MRI and BR1 CEUS findings. Case Rep Radiol 2012; 2012: 685486.

86. Moucari R, Rautou PE, Cazals-Hatem D, et al. Hepatocellular carcinoma in Budd-Chiari syndrome: Characteristics and risk factors. Gut 2008; 57: 828-835.

87. Ren W, Qi X, Yang Z, et al. Prevalence and risk factors of hepatocellular carcinoma in Budd-Chiari syndrome: A systematic review. Eur J Gastroenterol Hepatol 2013; 25 : 830-841.
88. Havlioglu N, Brunt EM and Bacon BR. Budd-Chiari syndrome and hepatocellular carcinoma: A case report and review of the literature. Am J Gastroenterol 2003; 98: 201-204.

89. Shin SH, Chung YH, Suh DD, et al. Characteristic clinical features of hepatocellular carcinoma associated with Budd-Chiari syndrome: Evidence of different carcinogenic process from hepatitis B virus-associated hepatocellular carcinoma. Eur J Gastroenterol Hepatol 2004; 16 : 319-324.

90. Yang $\mathrm{C}, \mathrm{Xu} \mathrm{K}$, Zheng J, et al. Hepatocellular carcinoma in Budd-Chiari syndrome: Enhancement patterns at dynamic gadolinium-enhanced T1-weighted MR imaging. Cell Biochem Biophys 2014; 70: 661-666. 